\title{
Value-Based Algorithms-The Key to Improving Patient Care
}

\author{
Sanjit R Konda*, Ariana Lott and Kenneth A Egol \\ Department of Orthopaedic Surgery, USA \\ *Corresponding author: Sanjit R Konda, Department of Orthopaedic Surgery, USA
}

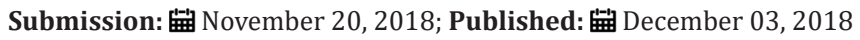

\section{Introduction}

Health Care costs in the United States are increasing exponentially with spending reaching 3.3 trillion dollars in 2016 [1]. Estimates show that the total spending on musculoskeletal care for patients over the age of 65 increased from $\$ 153$ billion between 1996 to 1998 to \$294 billion between 2009 and 2011 [2]. In light of these rising health care costs, many are developing strategies to improve quality by reducing the financial burden of these patients on the health care system while improving outcomes. This need has only increased in light of the recent transition from fee-for-service to bundle payment models of care and introduction of alternative payment models for orthopedic care by both the Centers for Medicare and Medicaid (CMS) and private payers. In order provide high-quality care and improve patient outcomes, it is essential to have an effective risk stratification tool to provide targeted resource-conscious and high-value care to both prototypical and outlier patients. The authors have published on the development of a novel inpatient mortality risk tool, the Score for Trauma Triage in the Geriatric and Middle-Aged (STTGMA).

This score, which evaluates a patient's age, medical comorbidities, vital signs, and anatomic injuries, has been validated in the National Trauma Databank as well as prospectively at an academic medical center and Level 1 Trauma Center as a reliable tool for triage analysis $[3,4]$. While originally designed to predict risk of inpatient mortality, it has also demonstrated usefulness in risk stratifying patients based on hospital quality measure outcomes and inpatient hospitalization cost. These hospital quality measures include length of stay, complications, need for intensive care unit level care, discharge disposition, and readmissions [5-7] One of the many benefits of this tool is its ability to be calculated quickly at the time of presentation in the emergency department even prior to admission to the hospital. The STTGMA score can be used to risk stratify all middle-aged and geriatric orthopedic trauma patients; however, particular attention was paid to hip and femur fracture patients given the inclusion of hip fracture patients treated with arthroplasty procedures in the current CJR bundle and inclusion of all hip and femur fractures that undergo operative fixation in the BPCI Advanced program [7,8]. By creating risk cohorts (minimal-, low-, moderate-, and high-risk), we were able to identify those patients who are outliers with regards to length of stay, complications, and resource utilization. A hip fracture specific novel cost-prediction tool was also created using similar variables to the STTGMA score to predict patients who were high-risk to have admissions with costs in the top 5\% [9]. By stratifying patients both by risk and cost, one can create a group of patients that should have similar outcomes with respect to risk and cost profiles. Any deviation from the mean outcome would signify a lower or higher quality outcome and any deviation from the mean cost would signify a lower or higher cost of care. Therefore, the overall value of the admission (quality=outcome/cost) could be calculated and compared to the baseline norms established via the novel valuebased algorithm. With its ability to risk stratify patients at the time of presentation, the STTGMA tool is the only available guide to clinical decision making that we know of that identifies high-risk patients at the onset of care and devoting more resources to these patients in a proactive rather than reactive fashion.

Additional resources such as case managers or specialized nurses can be assigned to these patients at the time of admission to increase the quality of these admissions. In addition to focusing on high risk STTGMA score patients, there are also opportunities to increase the quality of care for low risk STTGMA score patients. Identifying a patient as low-risk for complications and mortality at the onset of admission provides hospitals with opportunities to develop lower resource care pathways for these patients thereby improving the overall value of the care provided.

\section{Conclusion}

In the current health care climate, it is necessary for hospitals and physicians to develop and utilize tools that can provide more high-value care. Value-based algorithms, such as STTGMA, can serve as guides for inpatient triage towards specialized pathways of care to improve quality and decrease overall cost of care. These algorithms will provide the necessary information to make informed choices about complex health care decisions necessary in providing high quality care and creating high reliability organizations.

\section{References}

1. NHE Fact Sheet (2018) Centers for medicare and medicaid services.

2. (2018) United states bone and joint initiative: The burden of musculoskeletal diseases in the united states (BMUS), ( $3^{\text {rd }}$ edn), 2014. Rosemont, USA. 
3. Konda SR, Seymour R, Manoli A, Gales J, Karunakar MA (2016) Carolinas trauma network research. development of a middle-age and geriatric trauma mortality risk score: A tool to guide palliative care consultations. Bull Hosp J Dis 74(4): 298-305.

4. Konda SR, Lott A, Selah H, Schubl S, Robitsek J (2017) How does frailty factor into mortality risk-assessment of a middle-aged and geriatric trauma population? Geriatr Orthop Surg Rehabil 8(4): 225-230.

5. Konda SR, Lott A, Saleh H, Lyon T, Egol KA (2018) A novel tool to predict inpatient triage and hospital quality measures in the geriatric trauma population at time of admission. Presented as podium presentation at the american academy of orthopaedic surgeons annual meeting.
6. Konda SR, Lott A, Saleh H, Gales J, Egol KA (2018) Use of the STTGMA Tool to risk stratify 1 -year functional outcomes and mortality in geriatric trauma patients. J Orthop Trauma 32(9): 461-466.

7. Konda SR, Lott A, Egol KA (2018) The coming hip and femur fracture bundle: A new inpatient risk stratification tool for care providers. Geriatr Orthop Surg Rehabil.

8. Centers for Medicare \& Medicaid Services (2018) BPCI Advanced.

9. Konda SR, Lott A, Egol KA. Development of a value-based algorithm for inpatient triage of elderly hip fracture patients.
Creative Commons Attribution 4.0

International License

For possible submissions Click Here

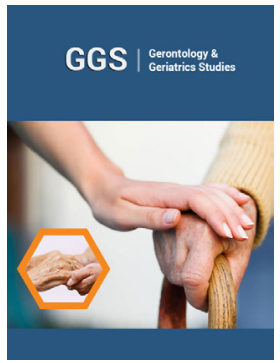

\section{Gerontology \& Geriatrics Studies}

Benefits of Publishing with us

- High-level peer review and editorial services

- Freely accessible online immediately upon publication

- Authors retain the copyright to their work

- Licensing it under a Creative Commons license

- Visibility through different online platforms 